\title{
Uncertainty propagation in a multiscale model of nanocrystalline plasticity
}

\author{
M. Koslowski ${ }^{\dagger *}$ and Alejandro Strachan ${ }^{\ddagger \dagger}$ \\ ${ }^{\dagger}$ School of Mechanical Engineering, \\ $\ddagger$ School of Materials Engineering and Birck Nanotechnology Center \\ Purdue University \\ West Lafayette, IN 47907, USA
}

December 1, 2010

\begin{abstract}
We characterize how uncertainties propagate across spatial and temporal scales in a physicsbased model of nanocrystalline plasticity of fcc metals. Our model combines molecular dynamics (MD) simulations to characterize atomic level processes that govern dislocation basedplastic deformation with a phase field approach to dislocation dynamics (PFDD) that describes how an ensemble of dislocations evolve and interact to determine the mechanical response of the material. We apply this approach to a nanocrystalline Ni specimen of interest in micro-electromechanical (MEMS) switches. Our approach enables us to quantify how internal stresses that result from the fabrication process affect the properties of dislocations (using MD) and how these properties, in turn, affect the yield stress of the metallic membrane (using the PFMM model). Our predictions show that, for a nanocrystalline sample with small grain size $(4 \mathrm{~nm})$, a variation in residual stress of $20 \mathrm{MPa}$ (typical in today's microfabrication techniques) would result in a variation on the critical resolved shear yield stress of approximately $15 \mathrm{MPa}$, a very small fraction of the nominal value of approximately $9 \mathrm{GPa}$.
\end{abstract}

\section{Introduction}

Multiscale materials models, where first principles-based atomic simulations inform mesoscale or macroscopic models enable predictive simulations of materials-specific processes and properties.

*marisol@purdue.edu

${ }^{\dagger}$ strachan@purdue.edu 
These models are particularly useful in areas where experiments are difficult and/or expensive to perform (such as micro- and nano-devices or for materials under extreme conditions) or to explore new materials not yet fabricated as part of materials design and optimization efforts. While multiscale materials modeling is playing an increasingly important role in many defense and commercial applications the accuracy of its predictions needs to be rigorously quantified before it is widely adopted as central tool in materials optimization and certification.

One of the main goals of first principles-based multi-scale approaches is to reduce the empiricism in material models by limiting the experimental inputs to information unrelated to the properties or process one intends to describe. For example, the initial composition and microstructure of a material should be obtained from experiments if fabrication and processing are not modeled. Assessing the accuracy and range of applicability of current state of the art multi-scale models against appropriate experiments remains a significant challenge that will not be fully addressed until uncertainty quantification (UQ) is performed both in the model and in experiments $[1 ; 2 ; 3]$. UQ is an intricate part of the field of Quantification of Margins and Uncertainties (QMU), a methodology to quantify design margins and uncertainties in a system in order to define confidence regions used to inform decisions in the certification of materials, structures, devices and engineering systems in general [2].

In this paper we present our work on UQ in a multiscale model for the mechanical response of of nanocrystalline Ni as part of Purdue's Center for the Prediction of Reliability, Integrity and Survivability of Microsystems (PRISM), part of the US Department of Energy NNSA Predictive Science Academic Alliance Program. The PRISM Center focuses on predicting the performance and reliability of RF-MEMS switches and the work presented here focuses on the electro-deposited Ni membrane of the switch. This is a challenging task both from the point of view of materials modeling and also in UQ due to the complex nanostructure of the materials involved and to the large variability typically observed in microdevices.

Physics-based predictive models for the mechanical response of micron and sub-micron sized specimens are very important in the fields of micro-electromechanical systems (MEMS) and microelectronics where components are subject to thermal and mechanical loads over extendend periods of time and experimental characterization is very challenging due to the dimensions involved. The reliability of the RF-MEMS switch of interest, see Figure 2.1 is strongly related to the microstructure of the device components including that of the Ni membrane (yellow in Fig. 2.1). The membrane moves down an contacts a dielectric pad when the switch is turned off via electrostatic actuation; when the voltage is remove the membrane returns to its original state driven by the elastic restoring force. Plastic deformation of the membrane affects the operation of the device affecting the pull-in (closing) and pull-out (opening) voltages; in fact, one of the main failure mechanisms of these devices is associated with creep deformation of the membrane [4]. Also, plastic deformation is critical to understand device survivability under extreme conditions, e.g. shock loading [5]. Device to device variability stemming from the fabrication procedure play a significant role in their reliability. In the present work we concentrate on the effect of the uncertainty in residual stress along the longitudinal direction of the membrane on its critical resolved shear stress (CRSS) 
of the membrane.

Plastic deformation, including creep, in these materials remain poorly understood. The main challenge being that their mechanical response depends very strongly on the characteristic size of their micro- or nano-structure (e.g. grain size and orientation) $[6 ; 7 ; 8 ; 9 ; 10 ; 11]$ and its size distribution $[12 ; 13 ; 14]$. These size effects arise from the the interplay between competing mechanisms of plastic deformation and the interaction between the defects responsible for them with each other and with the specimen boundaries. Thus, in order to capture such sub-continuum effects, detailed models describing individual deformation mechanisms are required. In this work we use a phase field approach to dislocation dynamics (PFDD) $[15 ; 16$; 17] where individual dislocations are explicitly described and molecular dynamics (MD) simulations to inform the PFDD with material specific properties of individual dislocations.

As mentioned above, UQ is particularly important in modeling and experiments of MEMS devices given the large variabilities in response observed in these devices even when built under nominally identical conditions using state of the art techniques [9; 18]. Relatively small changes in processing conditions lead to variabilities in size or microstructure that can often lead to significant changes in response. In this paper we focus on how the residual stress that develops in the metallic bridge of the MEMS switch during fabrication affects its plastic deformation that cause performance deterioration and even failure.

A second challenge in the quantification of uncertainties in multiscale models is the need to propagate them across models and scales. Here we quantify how residual stress (obtained experimentally) affects dislocation properties using MD simulations and how the resulting uncertainty in dislocation properties affects the plastic deformation of nanoscale polycrystals using the mesoscale PFDD model.

The paper is organized as follows. In Section 2 we describe the application of interest and our multiscale approach for crystal plasticity with an enphasis on how the PFDD parameters are obtained from MD simulations. Section 3 describes how aleatoric uncertainties are propagated from the finer scales (MD) to the coarse model (PFDD) and we show an example of how uncertainties inherent in the fabrication process are propagated across scales and affect the plastic deformation, in particular the yield stress. Finally conclusions are drawn in Section 4.

\section{Multiscale model of single crystal plasticity}

Figure 2.1 shows, schematically, the capacitance RF MEMS switch of interest. It consists of a nanocrystalline Ni membrane, shown on top, with the following approximate dimensions, length $l=400 \mu \mathrm{m}$, width $w=100 \mu \mathrm{m}$ and thickness $t=2 \mu \mathrm{m}$. When the contact is open a gap between the metallic membrane and dielectric pad leads to a very low capacitance, this is the on state of the switch as a RF signal in the metallic line below the dielectric will pass. The switch is turned to the off position by electrostatically actuating the metallic membrane and closing the gap that separates

it from the dielectric; the capacitance then increases by several orders of magnitude. The metallic 
membrane of RF MEMS is fabricated by electroplating which results in a complex microstructure with a grain size distribution from few nanometers to hundreds of nanometers [19]. Plastic deformation of such specimens, including creep, depend very strongly on this microstructure and differ significantly from bulk polycrystalline samples. Furthermore, residual stresses develop during fabrication, and while tensile stress is preferable for operation, this is not always achieved and fabrication results in residual stresses that vary from device to device. In this paper we propose a model for dislocation based plasticity inside the nanoscale grains and quantify how internal strain affects the the CRSS on the slip planes of each grain.

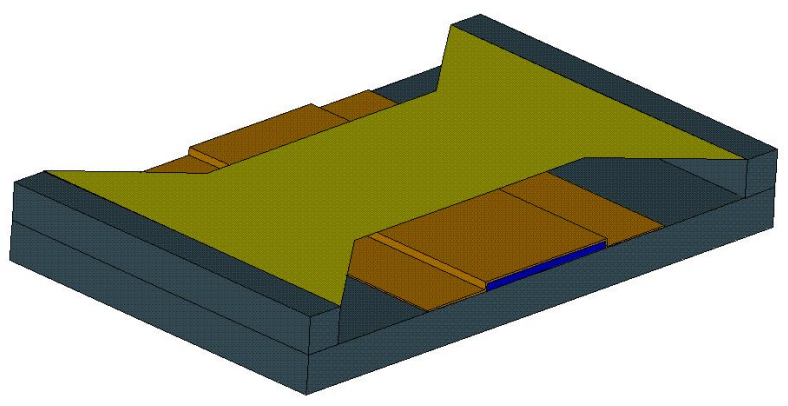

Figure 2.1: Schematic representation of the RF MEMS device

\subsection{Phase field dislocations model}

We use a phase field approach to study dislocation dynamics inside each grain. In this model, a phase field is defined for each slip system and its value indicates the relative displacement of the crystalline material on either side of the plane. The phase field is integer valued and, as Figure 2.2 illustrates, contour plots of the phase field represent dislocations. In fcc materials there are 12 slip systems and we represent the dislocation ensemble on each slip sytem by a separate phase field $\xi^{\alpha}(\boldsymbol{x})$ with $\alpha=1,2, \ldots 12$. In order to describe the time evolution of each phase field $\xi^{\alpha}(\boldsymbol{x})$ under possible applied external loads an expression is needed for the total energy $E[\boldsymbol{\xi}]$ and a system of coupled time dependent Ginzburg-Landau equation is solved:

$$
\frac{\partial \xi(\alpha, \boldsymbol{x})}{\partial t}=-L \frac{\partial E[\boldsymbol{\xi}]}{\partial \xi(\alpha, \boldsymbol{x})}
$$

where $\boldsymbol{\xi}$ represents all the phase fields, $\xi^{1}(\boldsymbol{x}), \xi^{2}(\boldsymbol{x}), \ldots, \xi^{12}(\boldsymbol{x})$. Therefore 2.1 represents a system of 12 coupled equations. The total energy of the phase field should take into account: i) the strain fields caused by each dislocation that governs their self-energies and long range interactions, ii) the energy near the dislocation line where atomic displacements are large enough that elasticity cannot be applied (i.e. the so called core energy), and, iii) the coupling with external mechanical loads. In the following sections we describe each of the energy terms. 


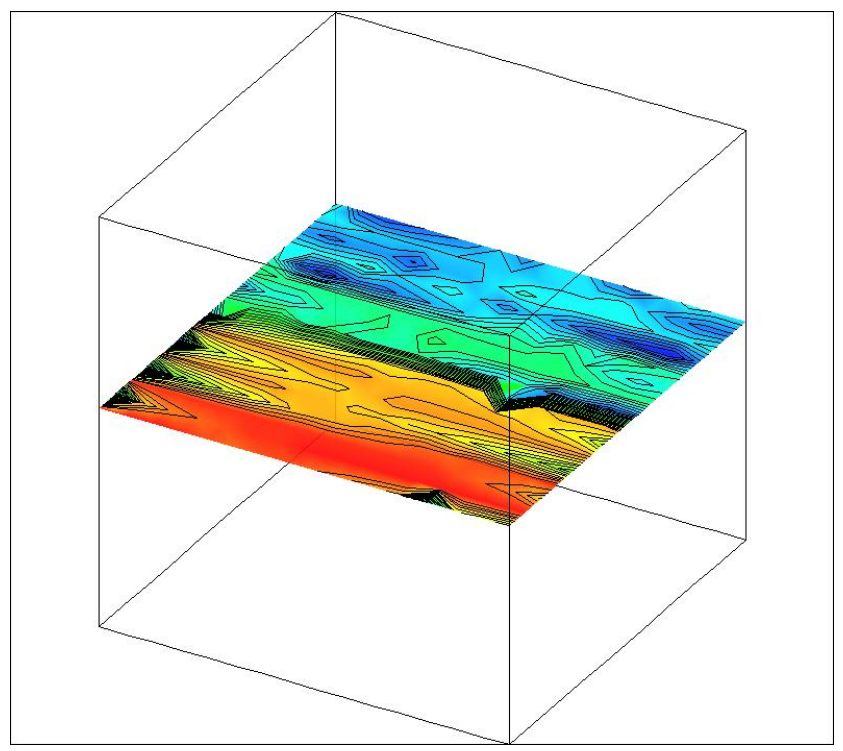

Figure 2.2: Dislocation lines represented as contours of the phase field in one active slip system

\subsubsection{Elastic energy}

The elastic energy can be written as [15]

$$
E^{\text {elas }}=E^{\text {dis }}+E^{\text {ext }}=\frac{1}{2} f \hat{A}_{m n u v}(\boldsymbol{k}) \hat{\beta}_{m n}^{p}(\boldsymbol{k}) \hat{\beta}_{u v}^{p *}(\boldsymbol{k}) \frac{d^{3} k}{(2 \pi)^{3}}-\int_{V} \sigma_{i j}^{e x t} \beta_{i j}^{p} d^{3} x
$$

where, here and subsequently, a superposed $(c)$ denotes the Fourier transform of a function, and

$$
\hat{A}_{\text {mnuv }}(\boldsymbol{k})=c_{\text {mnuv }}-c_{k l u v} c_{i j m n} \hat{G}_{k i}(\boldsymbol{k}) k_{j} k_{l}
$$

In this expression, $\boldsymbol{G}(\boldsymbol{x})$ is the Green's tensor of linear elasticity [20], $\hat{\boldsymbol{G}}(\boldsymbol{k})$ its Fourier transform, $\beta^{p}$ is the plastic distortion and $\sigma_{i j}^{e x t}$ is the externally applied stress. The first term in equation 2.2, $E^{\text {dis }}$, represents the elastic energy of the dislocations, while the second term, $E^{\text {ext }}$, represents the interaction with an external applied stress field.

Here we assume that plastic slip is confined to families of parallel slip planes in a crystal structure. Therefore:

$$
\beta_{i j}^{p}(\boldsymbol{x})=\sum_{\alpha=1}^{N} \sum_{n_{\alpha}=-\infty}^{\infty} \xi_{n_{\alpha}}^{\alpha}(\boldsymbol{x}) \delta_{n_{\alpha}} m_{i}^{\alpha} b_{j}^{\alpha}
$$

where $\alpha$ represents the slip plane family determined for the direction of the burgers vector $\boldsymbol{b}^{\alpha}$ and the normal to the slip plane $\boldsymbol{m}^{\alpha}$ and $\delta_{n_{\alpha}}$ is a Dirac distribution supported on the slip plane $n_{\alpha}$ and $N$ is the number of these families, 12 for fcc crystals. In equation 2.4 the sum of the slip, 
$\xi_{n_{\alpha}}^{\alpha}(\boldsymbol{x})$, over all the planes in a family $\alpha$ can be replaced by $\frac{1}{d} \xi^{\alpha}(\boldsymbol{x})$ where $d$ is the interplanar distance.

$$
\beta_{i j}^{p}(\boldsymbol{x})=\frac{b}{d} \sum_{\alpha=1}^{N} \xi^{\alpha}(\boldsymbol{x}) m_{i}^{\alpha} s_{j}^{\alpha}
$$

where $\boldsymbol{s}^{\alpha}$ is the direction of the burgers vector $\boldsymbol{b}^{\alpha}$ and $\frac{1}{d} \xi^{\alpha}(\boldsymbol{x})$ represents a three dimensional density of slip. Replacing 2.5 in 2.2 we obtain

$$
E^{i n t}=f \sum_{\alpha=1}^{N} \sum_{\alpha^{\prime}=1}^{N} \hat{B}_{\alpha \alpha^{\prime}}(\boldsymbol{k}) \hat{\xi^{\alpha}}(\boldsymbol{k}) \hat{\xi}^{\prime}(\boldsymbol{k}) \frac{d^{3} k}{(2 \pi)^{3}}
$$

where

$$
\hat{B}_{\alpha \alpha^{\prime}}(\boldsymbol{k})=\frac{b^{2}}{d^{2}} \hat{A}_{m n u v}(\boldsymbol{k}) m_{m}^{\alpha} s_{n}^{\alpha} m_{u}^{\alpha^{\prime}} s_{v}^{\alpha^{\prime}}
$$

\subsubsection{Core Energy}

The core energy represents the strain energy in atoms near the dislocation line where elasticity theory is not applicable due to the large distortions from the equilibrium structure. The first term used to describe such region associated with crystal disregistry takes into account that the energy of the crystal should attain a minima when the displacement jump $\xi(\boldsymbol{x}) b$ is an integer multiple of the Burgers vector, i.e. $\xi(\boldsymbol{x})$. Several potentials satisfy this condition, in the past we have used a piecewise quadratic potential [15]. Here we take this potential with a sinusoidal shape [21; 22]. In the present model we consider a crystal disregistry potential for each slip plane of the form

$$
\phi_{n_{\alpha}}(\boldsymbol{x})=E_{u s f}^{\alpha} \sin ^{2}\left(\pi \xi_{n_{\alpha}}^{\alpha}(\boldsymbol{x})\right) \delta_{n_{\alpha}}
$$

where $E_{u s f}$ is the unstabe stacking fault energy. The energy is therefore:

$$
\sum_{\alpha=1}^{N} \sum_{n_{\alpha}=-\infty}^{\infty} \int_{S_{n_{\alpha}}} \phi_{n_{\alpha}}(\boldsymbol{x}) d S
$$

where the integral is over each slip plane $n_{\alpha}$. Proceeding as in the previous section we replace the sum of the integral over all slip planes for a three dimensional integral and we obtain

$$
E^{\text {cryst }}=\frac{1}{d} \sum_{\alpha=1}^{N} E_{u s f}^{\alpha} \int \sin ^{2}\left(\pi \xi^{\alpha}(\boldsymbol{x})\right) d^{3} x
$$

The unstable stacking fault energy, $E_{u s f}$, is a key characteristic of the crystal disregistry potential and can be computed from atomistic simulations by sliding two perfect crystals with respect to 
each other. As a first approximation we can also estimate $E_{u s f}$ from the strain energy when a shear deformation is applied to the volume, in this case

$$
E_{u s f}=\frac{\mu b^{2}}{d \pi^{2}}
$$

where $\mu$ is the shear modulus. Replacing in 2.11 material constants of Ni single crystals $\mu=$ $97.3 G P a$ and considering a distance between active slip planes of $d=3 b$ we obtain $E_{u s f}=$ $0.50 \mathrm{~J} / \mathrm{m}^{2}$.

The crystal disregistry term above does not describe completely the energy associated with the dislocation's cores which is associated, within the phase field description, with gradients. This is a local energy and should describe the local strain energy inside a small region near the dislocation line. From elastic considerations this energy for a Peierls dislocation is [22]

$$
E^{\text {Peierls }}=\frac{\mu b^{2}}{4 \pi(1-\nu)}
$$

for an edge dislocation and

$$
E^{\text {Peierls }}=\frac{\mu b^{2}}{4 \pi}
$$

for a screw dislocation. This local energy can be taken into account with a local potential of the form:

$$
\psi\left(\xi^{\alpha}, \xi^{\beta}\right)=H(\alpha, \beta)_{i j k l} b_{i} \frac{\partial \xi^{\alpha}(\boldsymbol{x})}{\partial x_{j}} b_{k} \frac{\partial \xi^{\beta}(\boldsymbol{x})}{\partial x_{l}}
$$

As will be shown below, the dislocation core energy can be computed from atomistic simulations and these values will be used for our calculations.

The total core energy is therefore:

$$
E^{\text {core }}=\frac{E_{u s f}}{d} \sum_{\alpha=1}^{N} \int \sin ^{2}\left(\xi^{\alpha}(\boldsymbol{x})\right) d^{3} x+\sum_{\alpha \beta=1}^{N} \int H(\alpha, \beta)_{i j k l} b_{i} \frac{\partial \xi^{\alpha}(\boldsymbol{x})}{\partial x_{j}} b_{k} \frac{\partial \xi^{\beta}(\boldsymbol{x})}{\partial x_{l}} d^{3} x
$$

where again the value of the tensor $H(\alpha, \beta)_{i j k l}$ can be computed from atomistic simulations.

\subsubsection{CRSS calculations}

The CRSS computed from the 3D PFDD simulations is the quantity of interest to carry the UQ analysis under varying residual strains in the Ni membrane. In this section we compute the yield stress of a nanostructure consisting of a periodic arrangement of cubic grains with small dimensions ( $4 \mathrm{~nm}$ on the side) under monotonic loading. To simplify the analysis the loading condition is such that only one slip system is activated within the grains. The [111] direction of the fcc crystal 
is oriented along the $x_{3}$ direction and the active Burgers vector is in the $x_{1}$ direction. The system is then incrementally loaded with external stress $\sigma_{13}$, while the other components are set to zero.

The grain boundaries are represented by a distribution of dislocations from other slip systems (forest dislocations) that pierce the active slip plane and act as pinning points and sources of dislocations. This microstructure representing the grain boundaries is kept fixed throughout the simulations. The initial configuration for the PFDD simulations consists of a dislocation ensemble with density $\rho=10^{16} / \mathrm{m}^{2}$ and is otherwise defect free.

The materials parameters used in these simulations were obtained from MD simulations, as will be described bellow, and are shown in Table 1. The MD elastic constants for the material were converted to isotropic constants following Voigt's average [22] before being used in the PFDD simulations.

Figure 2.3 shows the stress-strain curve from the 3D PFDD simulations with an unstable stacking fault energy $E_{u s f}=0.56 \mathrm{~J} / \mathrm{m}^{2}$ a burgers vector $b=0.256 \mathrm{~nm}$ an initial dislocation density $\rho=10^{16} / \mathrm{m}^{2}$ and a slip plane distance $d=4 b$. Here, we define critical resolved shear stress for any of the [111] planes as the value at which the stress $\sigma_{13}$ yields. This stress is computed by the $0.2 \%$ offset method as shown in Figure 2.3. We obtain a CRSS $\sigma_{y}=9.25 G P a$ in very good agreement with atomistic simulations of nanocrystalline Ni by Farkas et al. [23] with similar sizes.

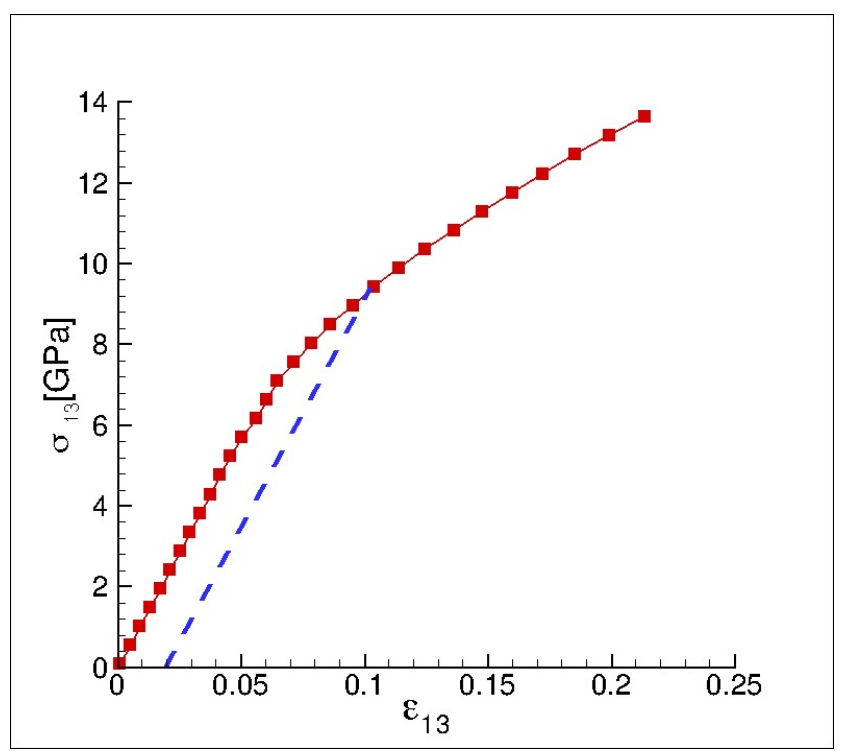

Figure 2.3: Stress strain curve of nanocrystalline Ni with $4 \mathrm{~nm}$. grain size

\subsection{Molecular dynamics}

In this section we briefly describe our MD simulations and how, from these simulations, we obtain materials parameters for the PFDD. MD describes the temporal evolution of a group of atoms 


\begin{tabular}{cccccc}
\hline \hline$c_{11}[G P a]$ & $c_{12}[\mathrm{GPa}]$ & $c_{44}[G P a]$ & $\lambda[G P a]$ & $\mu[G P a]$ & $\nu$ \\
\hline 225.37 & 156.92 & 97.3 & 118.0 & 58.38 & 0.334 \\
\hline
\end{tabular}

Table 1: Elastic constants

according to classical equations of motion:

$$
F_{i}=m_{i} \ddot{R}_{i}=\nabla E\left(\left\{R_{i}\right\}\right)
$$

where the index $i$ runs over all atoms, $F_{i}$ is the total force on atom $i, m_{i}$ is its mass and $R_{i}$ its position. Dots denote time derivative. In the absence of external fields the total force on an atom originates from the interaction with other (typically nearby) atoms. Atomic forces are obtained from the gradient of the total potential energy of the system with respect to atomic positions. This total energy can be obtained from first principles from a quantum mechanical calculation of the electronic structure of the system. However, ab initio electronic structure calculations remain computatioanlly intensive and in this paper we use an interatomic potential to describe atomic interactions, We use a many body embedded atom model (EAM) potential for $\mathrm{Ni}$, [24; 25] denoted quantum Sutton-Chen (qSC). The total energy within the EAM approximation is written as a sum of two-body terms plus the energy needed to embed each atom in the electronic density contributed by its neighbors:

$$
E\left(\left\{R_{i}\right\}\right)=\sum_{i<j} \phi\left(\left|R_{i}-R_{j}\right|\right)+\sum_{i} F\left(\rho_{i}\right)
$$

where the first sum runs over all pairs of atoms and $\phi$ denotes the two body interaction, the second sum runs over atoms, $F$ is the embedding energy and $\rho_{i}$ denotes the electron density at the location of atom $i$ contributed by its neighbors:

$$
\rho_{i}=\sum_{j \neq i} f\left(\left|R_{i}-R_{j}\right|\right)
$$

where $f(r)$ describes the electron density contribution of each atom. Note that if the embedding energy is non-linear the EAM energy expression cannot be written as a sum of pairwise terms.

\subsection{Informing the phase field model with atomistic information}

In the following subsections we describe how the parameters in the PFDD simulations are obtained from MD simulations. As mentioned above, there are three terms in the phase field energy expression. The terms associated with the elastic strain energy caused by the dislocations (including dislocation self energy and interactions) depend on elastic constants which are trivially obtained from the interatomic potential. The origin of this elastic energy and the core energy of the dislocations, described by Eq. 2.2 in the PFDD model, are described in the subsection 2.3.1. Finally, 
the term that describes the misfit energy associated with rigidly displacing two blocks of material across a plane, Eq. 2.10, is calculated from MD in subsection 2.3.2. Subsection 2.3.3 described PFDD simulations using this atomistic inputs.

\subsubsection{Atomistic simulations of dislocations}

The core energy energy term in the PFDD model, Eq. 2.2, associated with with the gradient of the phase field describes the strain energy near the dislocation line. This term, together with the crystal disregistry term addressed in the next section describes the energy of the dislocation core. As mentioned earlier, the total strain energy of the material with dislocations can be separated in two contributions: i) an elastic term that describes the dislocations self energy term and their interactions due to the long range deformation fields caused by them and, ii) the core energy: the energy near the dislocation line where atomic displacements are too large for elasticity theory to apply [22]. Thus, computing the core energy of dislocations requires an atomic description and in this sub-section we describe our approach.

We use zero temperature lattice parameter of 3.5064 Åto calculate dislocation core energies and characterize how strain affects the elastic energy. We start with a 6-atom unit cell with vectors $\mathrm{a}_{1}=1 / 2[112], \mathrm{a}_{2}=1 / 2[110]$ and $\mathrm{a}_{3}=[111]$ and replicate it 20 times along $\mathrm{a}_{1}, 7$ along $\mathrm{a}_{2}$ and 28 along $\mathrm{a}_{3}$ leading to a simulation cell with 23,520 atoms and lengths of $85.889 \AA, 17.356 \AA$, and 170.051 $\AA$. Periodic boundary conditions are imposed in all three directions. We then create a pair of screw dislocations with Burgers vectors $b=1 / 2[110]$ and $b=-1 / 2[110]$ using the atomic displacements from the elastic solution of the dislocations strain fields. Note that the total Burgers vector in the simulation cell is zero as is required by the periodic boundary conditions we impose. To obtaine the relaxed structure of the dislocation dipole we perform low temperature MD simulation ( 2 picoseconds at $\mathrm{T}=10 \mathrm{~K}$ ) followed by energy minimization using the qSC potential. Figure 2.4 shows an atomic snapshot of the relaxed configuration. As expected in fcc crystals each dislocation dissociates in two partial dislocations on a [111] plane; these partials are separated by a stacking fault made of hcp atoms (hcp atoms are shown as white spheres in Figure 2.4) (a).

Following our prior work, [26;27], in order to compute the elastic and core energies associated with the two dislocations we calculate the potential energy associated with each atom and compute their strain energy by subtracting the perfect crystal energy per atom. Figure 2.4) (b) shows the strain energy of the 400 highest energy atoms. We see a group of 168 atoms (12 per dislocation per Burgers vector) with an energy significantly higher than the rest, we define those atoms as the dislocation core. The core energy is simply the sum of the corresponding strain energies and we obtain a value of $0.49 \mathrm{eV} / \mathrm{b}$. The elastic strain energy is the sum of the strain energy of the remaining atoms in the simulation cell. The core energy reported here for Ni is very similar to prior calculations calculations also based on atomic simulations but using a different approach to differentiate elastic and core contributions [28]. 


\subsubsection{Lattice mismatch energy}

In order to compute the lattice mismatch energy, also known as gamma surface [29], we start with the 6-atom unit cell described above and a lattice parameter $a_{0}=3.524 \AA$. This lattice corresponds to zero pressure and $\mathrm{T}=300 \mathrm{~K}$ for the qSC potential without quantum corrections. The 6-atom unit cell is replicated 5 times along $\mathrm{a}_{1}, 8$ along $\mathrm{a}_{2}$ and 14 along $\mathrm{a}_{3}$ leading to a simulation cell 3,360 atoms. We impose periodic boundary conditions along the $\mathrm{a}_{1}$, and $\mathrm{a}_{2}$ directions and the system is divided in halves along the $\mathrm{a}_{3}$ direction. To compute the gamma surface the two halfves are rigidly displaced with respect to each other parallel to the $\mathrm{a}_{1}-\mathrm{a}_{2}$ planes in small increments and constraint molecular dynamics are performed for $15 \mathrm{ps}$ (the last $10 \mathrm{ps}$ of each run are used to compute the average energy). Atoms are constraint to moving only along the $\mathrm{a}_{3}$ direction to maintain the relative displacement between the two blocks. Also, atoms in the first and last unit cells along the $\mathrm{a}_{3}$ direction (240 atoms at each end) are fixed to their initial positions to avoid expansion normal to the (111) plane. Figure 2.5 (a) shows the resulting gamma surface as a function of displacement along the $<112>$ and $<110>$ directions obtained from our MD simulations.

In order to inform the PFDD we need the energy required to displace the half-crystals with respect the each other along the Burgers vector $(1 / 2[110])$. Figure 2.5 (b) shows the energy per unit area as a function of dispacement along $\mathrm{a}<110>$ direction obtained from the MD simulations (symbols) together with a fit of the first term of the core energy described in Equation 2.10 in the PFDD model; from this fit the parameter $E_{u s f}$ is obtained. We see that this parameter represents the so called unstable stacking fault energy, the maximum energy per unit area that occurs when the two crystals are displaced by half of a Burgers vector, or $1 / 4<110><110>$.

\section{Uncertainties and their propagation across scales}

There are a number of uncertain quantities in the prediction of plastic deformation of the nanocrystalline membrane of the RF MEMS device of interest. These uncertainties can be classified in two categories:

- Uncertainties in the Nickel membrane

- Residual stress developed during fabrication

- Grain size and crystal orientation distribution

- Variation in chemistry and composition (impurity atoms present predominantly in surfaces and grain boundaries)

- Membrane dimensions

- Uncertainties within the multiscale model

- MD input parameters (force field parameters) 
- FPDD input parameters (obtained from the MD simulations)

- Model form uncertainty both in MD and PFDD

The goal of this paper is not to fully characterize all uncertainties in the problem but to exemplify how uncertainties that originate from fabrication and that affect the setup and initial conditions of our MD simulations are propagated across scales and lead to uncertainties in our ultimate prediction: the CRSS predicted with the PFDD model. The important role of grain size and orientation distribution on mechanical response has been characterized extensively in the past and in this paper we focus on the role on uncertainties in residual stress. More specifically, we quantify how the residual stress experimentally measured in our RF switches lead to variations in the dislocation properties computed from MD and the CRSS predicted by the PFDD model for a given grain size and orientation.

A complete quantification of uncertainties of the mechanical response of the membrane requires, in addition, an experimental quantification of grain size and orientation distributions as well as a quantification of the variability of the level of impurities present in the device. These are aleatoric uncertainties that, at least in principle, are quantifiable experimentally. There are additional uncertainties associated with the models, including the force field parameters used in the MD simulations and model form uncertainties both at the atomistic and mesoscale scales. This last class of uncertainty originates either from lack of knowledge or a desire of computational efficiency and not from input parameters; for example: the functional forms of the MD potentials or the fact that the PFDD model does not describe the dissociation of dislocations into partials and does not allow for cross-slip.

The first step in the UQ process process is to characterize the response functions of each model to their uncertain input parameters: i) the CRSS predicted by the PFDD model as a function of unstable stacking fault energy and elastic energy of the dislocations; and ii) the effects on strain on the properties calculated using MD that are input the PFDD mode (unstable stacking fault, core energy and elastic energy)1. In Subsection 3.3 we use these response surfaces to predict how the uncertainties in macroscopic residual stress measured experimentally propagate across our models and lead to a probability density distribution (pdf) of CRSS.

\subsection{Sensitivity and response function of the PFDD model}

We carry out simulations with the PFDD where the unstable stacking fault energy is varied around its mean value, $<E_{u s f}>=0.56 \mathrm{~J} / \mathrm{m}^{2}$, as follows

$$
E_{u s f}=<E_{u s f}> \pm \Delta E_{u s f}
$$

with $\Delta E_{u s f} /<E_{u s f}>=0.05$. We also vary the elastic energy of the dislocations by multiplying the energy by a proportionality factor $\alpha$ to represent the effect of residual strains in the elastic dislocation energy

$$
E^{d i s}=\alpha E^{d i s}
$$


where $\alpha=\langle\alpha\rangle \pm \Delta \alpha$ with $\langle\alpha\rangle=1$ and $\Delta \alpha /<\alpha>=0.05$.

The PFDD simulations show that if the microstructure, including grain size and initial dislocation density, is kept fixed the unstable stacking fault energy, $E_{u s f}$ in the first term in Equation 2.10 and the elastic energy of the dislocations, first term in Equation 2.2, are the dominant parameters in determining the CRSS. The average values of $E_{u s f}$ and $E^{d i s}$ and their ranges are computed from atomisitics simulations as it will be shown in the next section.

The CRSS predicted by the PFDD model depends on grain size and orientation relative to the applied load, as well as the materials properties calculated from atomistic simulations. In the past we have studied the influence of thickness in passivated thin films [16;17] with results in good agreement with experiments. Here we characterize how the unstable stacking fault energy and the elastic energy that govern dislocation interactions and self energy affect the predicted CRSS for the nanocrystalline Ni model described in Section 2.1.3 .

Figure 3.1 shows the calculated response function predicted by the PFDD model for the CRSS as $E_{u s f}$ and the elastic dislocation energy are varied by 5\%. Fitting this curve to a linear response surface yields:

$$
\sigma_{Y}\left(E_{u s f}, \alpha\right)=9.28 G P a+3.63\left(\frac{E_{u s f}}{<E_{u s f}>}-1\right) G P a+0.07\left(\frac{\alpha}{<\alpha>}-1\right) G P a
$$

where $\left\langle E_{u s f}>\right.$ is the nominal value for the unstable stacking fault energy taken to be $0.56 \mathrm{~J} / \mathrm{m}^{2}$ and $\langle\alpha\rangle$ is taken as 1 with a correlation $R^{2}=0.96$.

Our results show that the CRSS depends most strongly on the unstable fault energy than on the elastic energy, the difference in normalized sensitivity is about a factor 50. As the unstable stacking fault energy increases a larger applied stress is needed to nucleate and move dislocations which in turns increases the yield stress.

\subsection{Sensitivity and response functions of molecular dynamics}

In this section we characterize how the internal strain affects the dislocation properties obtained from our MD simulations that inform the PFDD model. We characterize how the core energy, elastic energy of the set of dislocations and unstable stacking fault energy vary when a range of bi-axial strains within the slip plane ( $\mathrm{a}_{1}$ and $\mathrm{a}_{2}$ directions) and strains normal to it (along $\mathrm{a}_{3}$ ). Both the bi-axial in-plane strain and out-of-plane strain are varied between -0.01 and 0.01.

Figure 3.2 shows how the unstable stacking fault energy (a), core energy (b), and elastic energy (c) depend on the transverse and longitudinal strain. We see that the elastic energy of the dislocations vary by about $10 \%$ when the strain change by $\pm 1 \%$ while the properties associated with the dislocations core are slightly more sensitive to strain. Since our PFDD simulations show that the unstable stacking fault energy has the largest effect on the materials CRSS we focus on this response function [Fig. 3.2 (a)]. Fitting the MD data with a linear function on both strains leads to the following response surface as a function of the transverse and longitudinal strains: 


$$
E_{U S F}\left(\epsilon_{t}, \epsilon_{l}\right)=0.541 \mathrm{~J} / \mathrm{m}^{2}-1.67 \epsilon_{t} \mathrm{~J} / \mathrm{m}^{2}-4.75 \epsilon_{l} \mathrm{~J} / \mathrm{m}^{2}
$$

with a correlation $R^{2}=0.95$. This is an interesting result and quantifies how the unstable stacking fault energy decreases with tensile (positive) strain in either the transverse or longitudinal directions. Furthermore, our results show that strain normal to the sliding surface affects $E_{U S F}$ more significantly that strain within the surface. This result is not very surprising but our MD simulations provide a quantitative characterization.

\subsection{Uncertainty propagation}

With the various response functions at hand we can now propagate uncertainties in residual stress across the scales and quantify how they affect the CRSS. Since the PFDD shows the normalized sensitivity of the quantity of interest with respect to unstable staking fault energy is approximately 50 times larger that that for the elastic energy we use MD to predict how the strain on each of the nanocrystals of the Ni membrane affects $\mathrm{E}_{u s f}$ and use this information to predict a pdf of CRSS.

In order to estimate the distribution of strain on each of the 12 slip systems in each grain of the membrane we start with the experimentally derived residual stress distribution and use texture information obtained from X-ray diffraction experiments [19]. Alexeenko et al. characterized the residual stress from voltage-deflection measurements on 12 RF MEMS devices [, Alexeenko:2010] in these experiments the authors assume a Young's modulus for the membrane and obtain the residual stress in the axial direction of the membrane. For a Young's modulus E=200 GPa they obtain a mean residual of $25.25 \mathrm{MPa}$ with a standard deviation of 19.23 MPa. XRD experiments show that the Ni membrane of the device to have a strong [001] fiber texture due to preferential grain growth during electrodeposition. For this study we will simplify this microstructure and assume all grains are oriented with the [001] in the vertical direction (z axis) and are randomly orientated in the $x-y$ plane. In order to obtain the in-plane and out-of-plane strain distribution on the slips systems of the nanocrystalline membrane we follow the following three steps:

- Obtain a distribution of macroscopic residual stresses from the experimental pdf

- Compute the macroscopic strain tensor of the membrane using the Young's modulus used to extract the residual stress distribution and a Poisson ratio $\nu=0.31$.

- Generate a distribution of crystal orientations for each of the crystalline grains in the membrane and find the strain tensor in each of the 12 slip systems within the isostrain approximation (the strain in each grain, along the axes of the membrane, is equal to the macro-strain).

We generate an ensemble of 1000 residual stresses from the experimental distribution for E=200 GPa and, see Figure 3.3(a), representing 1000 membranes. For each macroscopic state we stochastically generate 5000 grain orientations and calculate the strain tensor in each of the 12 
slip systems of each grain. The pdf's of the two components of the in-plane strain (averaged over over two normal directions contained in the slip plane) and the two out-of-plane strain are shown in Fig. 3.3(b). Using the response function in Eq. 3.4 obtained from MD simulations we compute the pdf of the unstable stacking fault energies [3.3(c)]. Finally, we compute, for each slip system, its corresponding CRSS; the resulting pdf is shown in 3.3(d).

Our results show that residual stresses resulting from the fabrication on our RF MEMS devices has a small effect on the CRSS of the crystalline grains of the membrane. The width of the CRSS pdf depends on the distribution of residual stresses which in, in turn, are affected by the Young's modulus used in the experiments. However, for both values of the Young's moduli used (that represent a conservative bound on the possible values) the CRSS varies by no more $15 \mathrm{MPa}$, a very small fraction of the CRSS.

\section{Discussion and conclusions}

In this paper we propagate uncertainties across scales in a multi-scale model of single crystal plasticity. Our approach combines atomistic simulations to characterize materials properties associated with dislocations and elasticity with a phase field approach to dislocation dynamics. This is a powerful approach since atomistic simulations provides a first principles characterization of materials properties making it generally applicable and predictive and the phase field approach, where individual dislocations are described, captures how micro- or nanostructure and texture affects plasticity.

Such physics-based, predictive models of the mechanical response of metals are critical for a wide range of defense and commercial applications; this is particularly important for micro-scale specimens where experimental testing is challenging and also to understand materials response under extreme conditions of pressure and temperature. While significant progress has has been made in multiscale modeling in recent years, much work remains to be done in assessing the accuracy of the resulting predictions. Uncertainty quantification is a critical step in such validation efforts and this paper describes our efforts in this area.

Our approach to predict polycrystalline plasticity consists of two models that describe the material with different resolution. We use MD to quantify the atomic-level processes that govern dislocation-based plasticity in metals and a phase field approach to dislocation dynamics to predict the materials response governed by groups of dislocations evolving and interacting with one another. An important aspect of our approach is that every single material property used as input to the PFDD model is calculated from a MD simulation. In this paper we quantify how these materials properties depend on the residual strain of the individual grains in a nanocrystalline $\mathrm{Ni}$ membrane in an RF-MEMS switch. To achieve this we generate response functions from extensive MD simulations. We also quantified how these materials properties, in turn, affect the main prediction of the PFDD model, i.e. the critical resolved shear stress. These response functions enable us to propagate uncertainties across scales and quantify the role of residual strain on the 
yield stress of the metallic membranes. We find that, for nanocrystalline Ni with grain size of 4 $\mathrm{nm}$, the variability in residual stress measured in our device would lead to change in CRSS of 15 $\mathrm{MPa}$, a small fraction of the CRSS stress of approximately 9GPa. While our results show that the variability of residual stress due to fabrication does play an important role on determining the membrane strength, it can play an important role in other mechanical processes such as creep.

Significant work remains to be done in UQ of MD simulations and PFDD modeling. Despite some progress [30] one the key challenges in the area of MD are to estimate the uncertainty in the parameters used to describe the interactions between atoms and the functional forms themselves. In the area of PFDD we are currently working on the incorporation of partial dislocations in the description and in more accurate representations of grain boundaries. Addressing, epistemic uncertainties in this mesoscale model could be done by direct comparisons of PFDD simulations with large-scale MD where the exact same process is modeled.

\section{Acknowledgements}

The authors are grateful for support from the Purdue PRISM center under Department of Energy (National Nuclear Security Administration) award number DE-FC52-08NA28617. J. Murthy, D. Peroulis and A. Alexeenko are gratefully acknowledged for useful discussions and access to experimental data.

\section{References}

[1] J. C. Helton. Conceptual and computational basis for the quantification of margins and uncertainty. In Sandia Report SAND2009-3055. 2009.

[2] David H. Sharp and Merri M. Wood-Schultz. Qmu and nuclear weapons certification whats under the hood? Los Alamos Science, 28:47-53, 2003.

[3] Francois Hemez. Uncertainty quantification and the verification and validation of computational models. In D.J. Inman, C.R. Farrar, V. Lopes Jr., and V. Steffen Jr., editors, Damage Prognosis for Aerospace, Civil and Mechanical Systems. John Wiley and Sons Ltd., London, United Kingdom, 2003.

[4] Gabriel M. Rebeiz, editor. MEMS: theory, design and technology. John Wiley and Sons Inc., Hoboken, NJ, 1st edition, 2003.

[5] J. Kimberley, R. S. Cooney, J. Lambros, I. Chasiotis, and N. S. Barker. Failure of au rf-mems switches subjected to dynamic loading. Sensors and Actuators A, 154:140-148, 2009.

[6] R. Venkaltraman and C. Bravman. Separation of film thickness and grain boundary strengthening in al thin films on si. Journal of Materials Research, 7(8):2040-2048, 1992. 
[7] H. D. Espinosa, B. C. Prorok, and B. Peng. Plasticity size effects in free-standing submicron polycrystalline fcc films subjected to pure tension. Journal of the Mechanics and Physics of Solids, 52(3):667689, 2004.

[8] Y. Xiang, T. Y. Tsui, and J.J. Vlassak. The mechanical properties of freestanding electroplated cu thin films. Journal of Materials Research, 21(6):1607-1618, 2006.

[9] K.J. Hemker and W. N. Sharpe. Microscale characterization of mechanical properties. Annual Review of Materials Science, 37:93-126, 2007.

[10] T. G. Desai and P. M. Wolf. Is diffusion creep the cause for the inverse hall-petch effect in nanocrystalline materials? Materials Science and Engineering A, 493:41-47, 2008.

[11] B. A. Gruber, J. Bohm, F. Onuseit, A. Wanner, R. Spolenak, and E. Arzt. Size effects on yield strength and strain hardening fro ultra-thin cu films with and without passivation: A study by synchrotron and bulge test techniques. Acta Metallurgica, 56:2318-2335, 2008.

[12] J. Rajagopalan, J. H. Han, and T. A. Saif. Plastic deformation recovery in freestanding nanocrystalline aluminum and gold thin films. Science, 315:427-576, 2006.

[13] M. Koslowski. Effect of grain size distribution on plastic strain recovery. Physical Review B, 82:054110, 2010.

[14] R Spolenak, W. L. Brown, N. Tamura, A. A. MacDowell, R. S. Celestre, H. A. Padmore, B. Valek, J. C. Bravman, T. Marieb, H. Fujimoto, B. W. Batterman, and J. R. Patel. Local plasticity of al thin films as revealed by x-ray microdifraction. Physical Review Letters, 90(9):96102, 2003.

[15] M. Koslowski, A. Cuitiño, and M. Ortiz. A phase-field theory of dislocations dynamics, strain hardening and hysteresis in ductile single crystals. Journal of the Mechanics and Physics of Solids, 50(12):2957-2635, 2001.

[16] M. Koslowski. Scaling laws in plastic deformation. Philosophical Magazine, 87(8-9):1175-1184, 2007.

[17] A. Hunter and M. Koslowski. Direct calculation of material parameters for gradient plasticity. Journal of the Mechanics and Physics of Solids, 56:3181-3190, 2008.

[18] A. Alexeenko, S. Chigullapallia, J. Zeng, X. Guoa, A. Kovacs, and Peroulis D. Uncertainty in microscale gas damping: Implications on dynamics of capacitive mems switches. Reliability Engineering and System Safety, this issue, 2010.

[19] P. Cantwell and E. A. Stach. Private Communication, 2010.

[20] T. Mura. Micromechanics of defects in solids. Kluwer Academic Publishers, Boston, 1987.

[21] Y.U Wang, Y.M Jin, A.M. Cuitiño, and A. G. Khachaturyan. Phase field microelasticity theory and modeling of multiple dislocation dynamics. Applied Physics Letters, 78(16):2324-2326, 2001. 
[22] J. P. Hirth and J. Lothe. Theory of Dislocations. McGraw-Hill, New York, 1968.

[23] D. Farkas and B. Curtin. Plastic deformation mechanisms in nanocrystalline columnar grain structures. Materials Science and Engineering A, 412:316-322, 2005.

[24] Y. Kimura, Y. Qi, T. Cagin, and W.A. Goddard III. The quantum sutton-chen many-body potential for the properties of fcc metals. Unpublished.

[25] SN Luo, TJ Ahrens, T Cagin, A Strachan, WA Goddard, and DC Swift. Maximum superheating and undercooling: Systematics, molecular dynamics simulations, and dynamic experiments. PHYSICAL REVIEW B, 68(13), OCT 12003.

[26] GF Wang, A Strachan, T Cagin, and WA Goddard. Atomistic simulations of kinks in 1/2a $; 111 i$ screw dislocations in bcc tantalum. PHYSICAL REVIEW B, 68(22), DEC 2003.

[27] GF Wang, A Strachan, T Cagin, and WA Goddard. Molecular dynamics simulations of $1 / 2$ a(111) screw dislocation in Ta. MATERIALS SCIENCE AND ENGINEERING A-STRUCTURAL MATERIALS PROPERTIES MICROSTRUCTURE AND PROCESSING, 309(Sp. Iss. SI):133-137, JUL 152001. International Conference on the Fundamentals of Plastic Deformation, GAITHERSBURG, MARYLAND, JUN 19-22, 2000.

[28] Y Qi, A Strachan, T Cagin, and WA Goddard. Large scale atomistic simulations of screw dislocation structure, annihilation and cross-slip in FCCNi. MATERIALS SCIENCE AND ENGINEERING ASTRUCTURAL MATERIALS PROPERTIES MICROSTRUCTURE AND PROCESSING, 309(Sp. Iss. SI):156-159, JUL 152001.

[29] V VITEK. INTRINSIC STACKING FAULTS IN BODY-CENTRED CUBIC CRYSTALS. PHILOSOPHICAL MAGAZINE, 18(154):773-\&, 1968.

[30] S. L. Frederiksen, K. W. Jacobsen, K. S. Brown, and J. P. Sethna. Bayesian ensemble approach to error estimation of interatomic potentials. Physical Review Letters, 93(16):165501, 2004. 
(a)

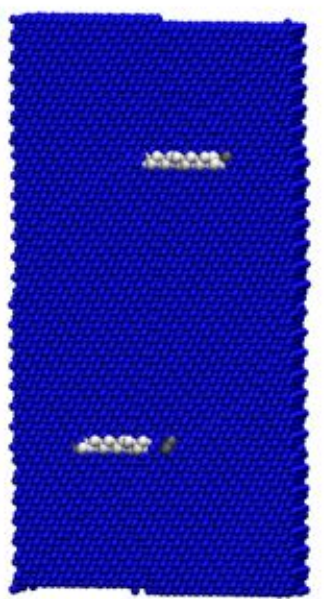

(b)

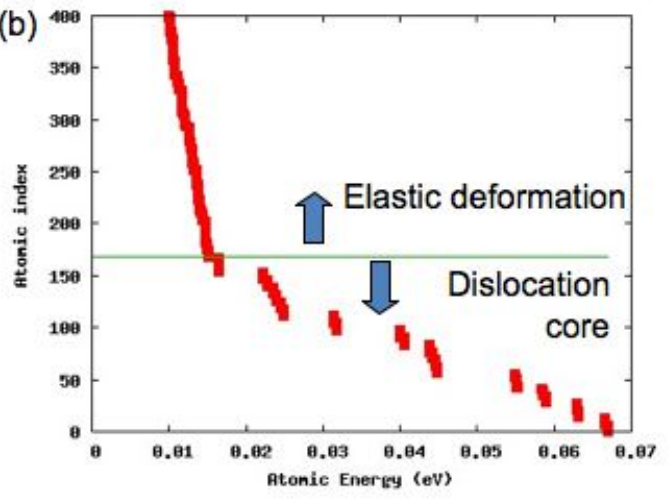

Figure 2.4: MD simulations of dislocations in Ni. (a) Atomic snapshot of two dissociated screw dislocations; light atoms denote stacking faults. (b) Atomic energy in decreasing order as a function of atom number for our relaxed simulation cell with two dissociated screw dislocations, each seven Burgers vector long; we define core as the 12 atoms with highest energy per dislocation, per Burgers vector. 

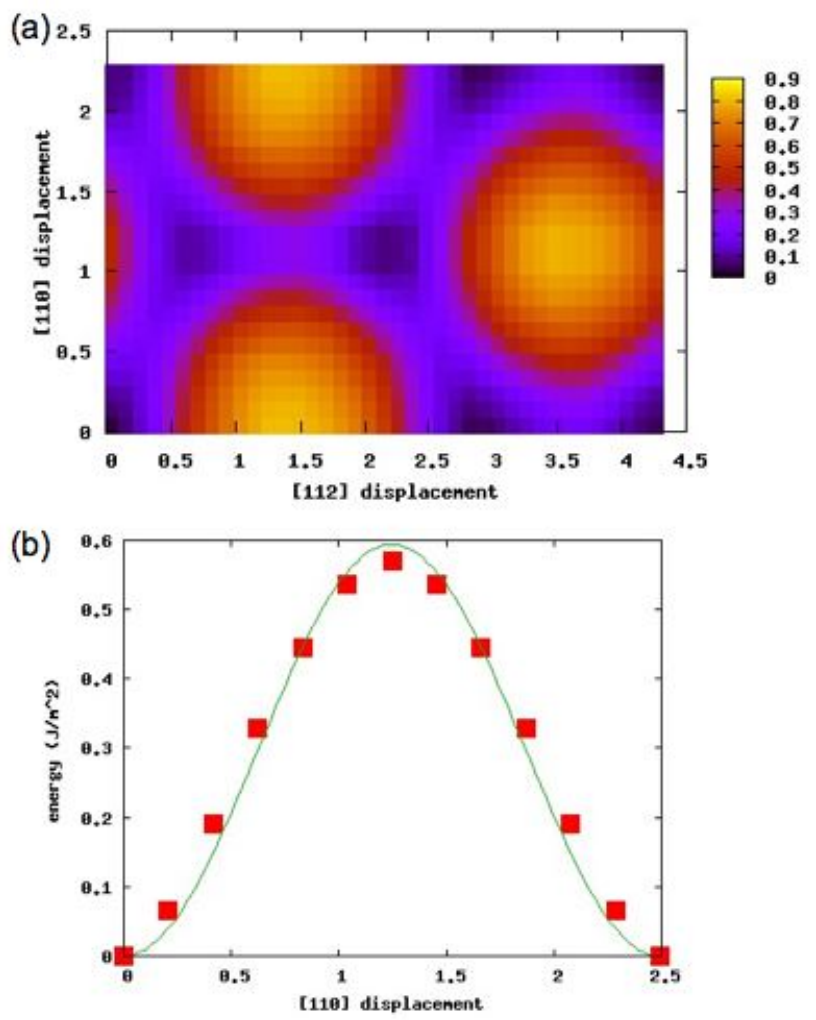

Figure 2.5: Gamma surface of $\mathrm{Ni}$ at $\mathrm{T}=300 \mathrm{~K}$. (a) 2D map of the energy per unit area as two blocks are displaced in the (111) plane. (b) Energy per unit area for a displacement along the [110] direction; squares show MD results and the line is a fit of the function used in the PFDD model to the MD data. 


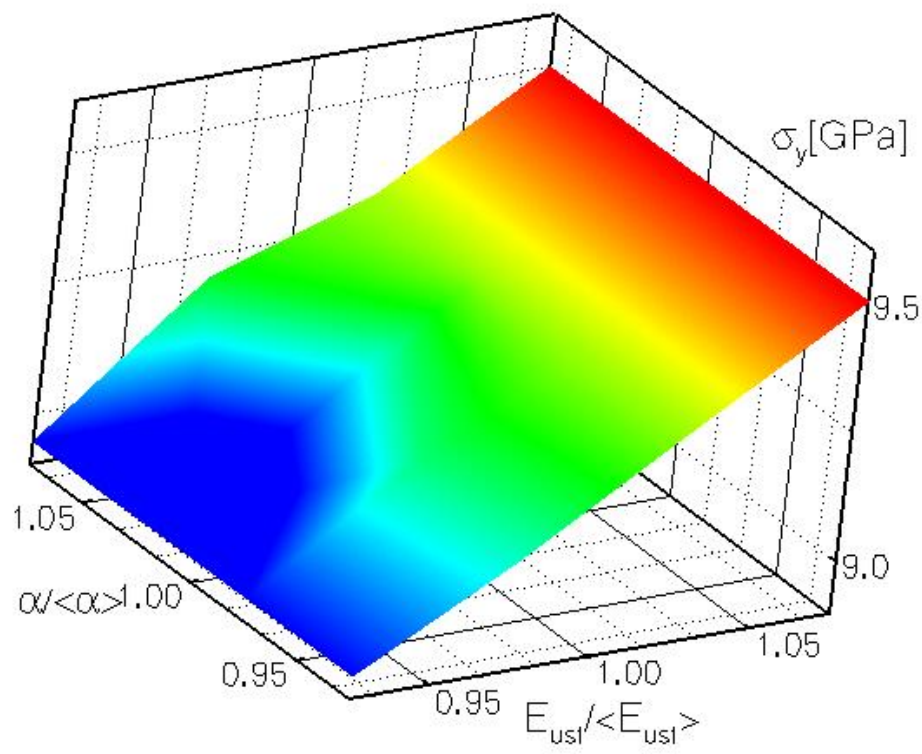

Figure 3.1: CRSS response function calculated from PFDD simulations as a function of the elastic energy and the unstable stacking fault energy. 


\section{(a) Unstable stacking fault $\left(\mathrm{J} / \mathrm{m}^{2}\right)$}

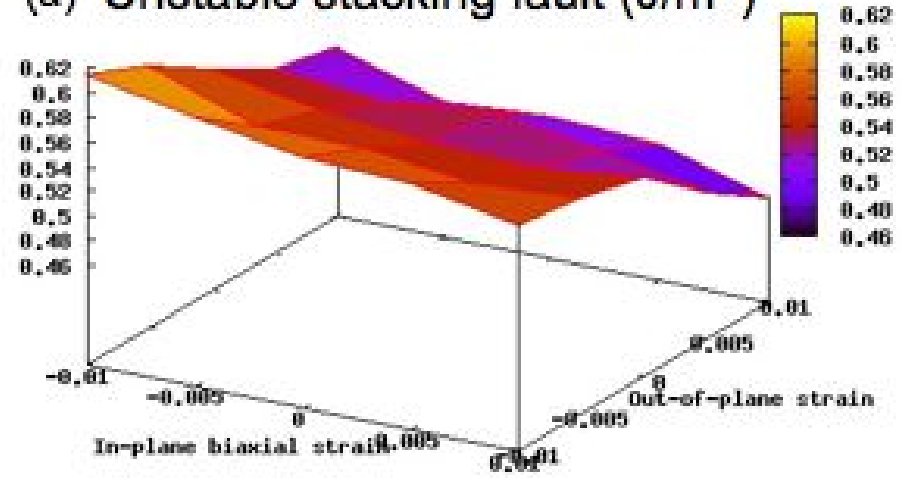

(b) Core energy (eV/b)
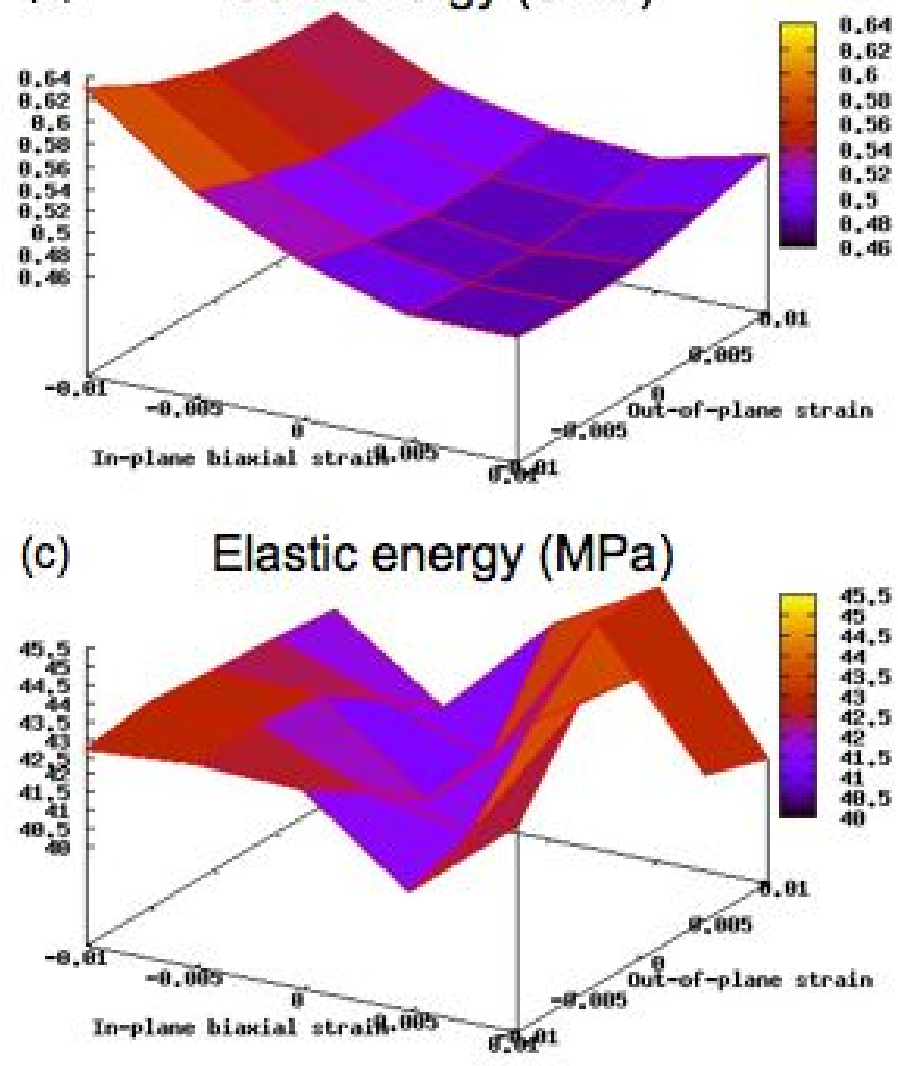

Figure 3.2: Response functions of MD parameters as a function of in-plan and out-of-plane strain. (a) Unstable stacking fault energy. (b) core energy. (c) elastic energy of dislocation system. 
(a)
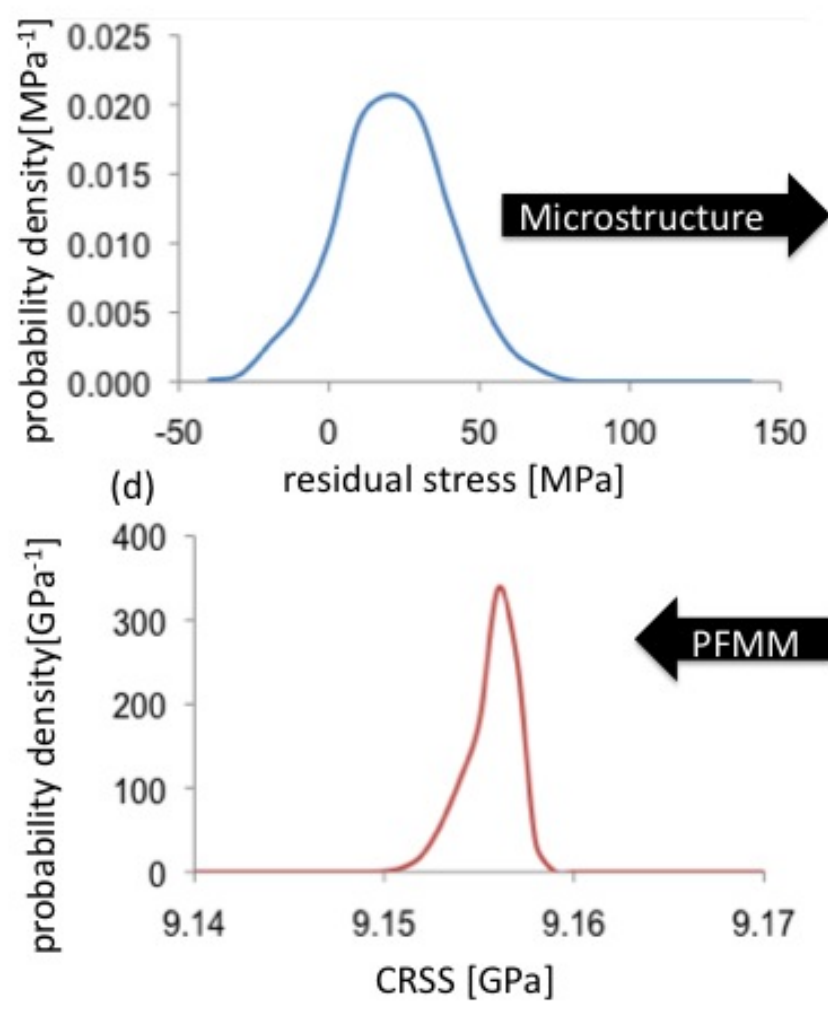

(b)
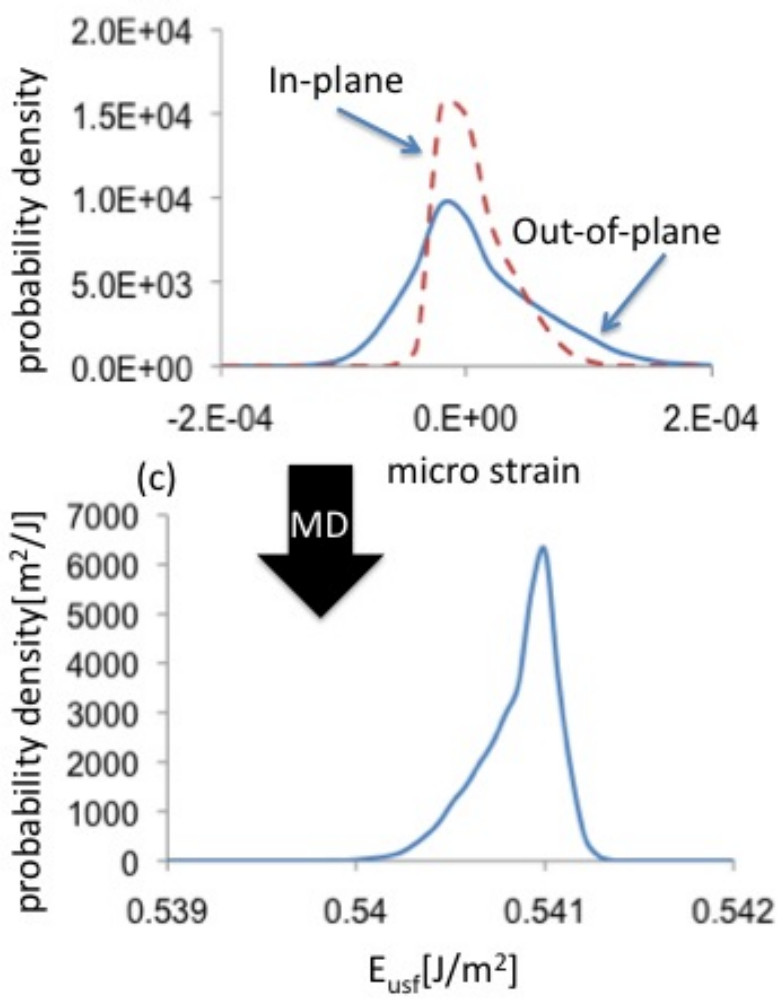

Figure 3.3: Uncertainty propagation across scales. The experimental distribution of residual stresses in the longitudinal direction of the membrane (a) is converted into a distribution of inplane and out-of-plane stress for each grain using the iso-strain approximation and XRD texture (b). The strains are then used with an MD response surface to predict the distribution of unstable stacking fault energies (c) that are used to predict a pdf of CRSS using PFMM response surfaces (d). 